\section{Mikrobiom normalisieren statt Atemwege von Keimen zu befreien}

Roquilly A et al. Pathophysiological role of respiratory dysbiosis in hospital-acquired pneumonia. Lancet Respir Med 2019; 7: 710 - 720

Die Inzidenz von im Krankenhaus erworbenen Pneumonien (hospitalaquired pneumonia, HAP) ist trotz bisheriger Maßnahmen anhaltend hoch. Nach neueren Erkenntnissen sind die Atemwege nicht steril, sondern auch im gesunden Zustand mikrobiell besiedelt. Möglicherweise bieten Ansätze zur Beeinflussung der Interaktion von Lungenmikrobiom und Immunsystem des Patienten Chancen, die Häufigkeit und Komplikationsrate von HAP zu senken.

Den Stand des Wissens hierzu hat eine internationale Wissenschaftlergruppe mit Antoine Roquilly, Mikrobiologe und Immunologe von der Universität Nantes in Frankreich, als Erstautor in einer Übersichtsarbeit zusammengefasst. Sie konstatiert, dass die Prävention und Behandlung der HAP basierend auf der Vorstellung der bakteriellen Kontamination ansonsten steriler Atemwege als Ursache verbesserungswürdig ist. Moderne Analysemethoden haben gezeigt, dass die Atemwege von einem Bakterien-Ökosystem hoher Diversität und Dynamik besiedelt sind, das beständig über die Mukosa mit dem Immunsystem des Wirts Mensch interagiert. Das verändert zwangsläufig auch das Verständnis der Pathophysiologie von Atemwegserkrankungen und der Pneumonie.

\section{Veränderungen bei HAP}

Bei mechanisch beatmeten Patienten lässt sich im Zeitverlauf eine Zunahme der mikrobiellen Biomasse feststellen. Gleichzeitig nimmt die Diversität des Mikrobioms schon vor der klinischen Entwicklung einer HAP ab. Um eine HAP zu verhindern oder zu behandeln, wäre daher ein neuer Ansatz, das Mikrobiom zu beeinflussen, anstatt es mit Antibiotika zu eliminieren. Auf der anderen Seite könnte es auch sinnvoll sein, auf das Immunsystem des Patienten einzuwirken: Studien haben gezeigt, dass bei stationärer Aufnahme von als immunkompentent eingestuften Patienten im Verlaufe der Immunosuppression bei kritischer Erkrankung substanzielle Veränderungen der Funktion verschiedener Immunzellen zu beobachten sind. Deshalb haben Therapien, die auf die Wiederherstellung der normalen Immunfunktion ausgerichtet sind und nicht direkt auf die Pathogene abzielen, das Potenzial, die Krankheitslast von HAP zu senken. Für die Prävention und Behandlung der HAP sollten demnach die Normalisierung der Diversität des Atemweg-Mikrobioms und die Wiederherstellung der mukosalen Immunität stärker im Vordergrund stehen statt der bisherigen Vorgehensweise, die Atemwege zu sterilisieren.

\section{FAZIT}

Die völlig neue Sicht auf die Physiologie der Atemwege mit einem Zusammenspiel von Mikrobiom und Wirtsimmunsystem könnte vielfältige Folgen für die Klinik haben, erläutern die Autoren: Die weitere Forschung könnte ganz neue Biomarker hervorbringen, ein neues Verständnis von Subtypen der HAP liefern und neue therapeutische Ansätze zur Beeinflussung des Mikrobioms und Immunsystems, beispielsweise durch gezielten Mikrobiomtransfer oder die Gabe von Immunmodulatoren, liefern.

Friederike Klein, München 